\title{
Osseous Metaplasia of the Transverse Humeral Ligament in a 10-Year-Old Bengal Cat
}

\author{
Verónica Rodiño Tilve ${ }^{1}$ Thomas Maddox ${ }^{2}$ Lorenzo Ressel ${ }^{3}$ Rob Pettitt ${ }^{2}$ \\ 1 Small Animal Surgery Department, Southfields Veterinary \\ Specialists, Basildon, Essex, United Kingdom \\ 2 Small Animal Teaching Hospital, Institute of Veterinary Science, \\ University of Liverpool, Wirral, United Kingdom \\ ${ }^{3}$ Department of Veterinary Pathology and Public Health, University of \\ Liverpool, Wirral, United Kingdom

\begin{abstract}
Address for correspondence Verónica Rodiño Tilve, BVSc, GPCert, $\mathrm{PgC}(\mathrm{SAS})$, Southfields Veterinary Specialists, 1 Bramston Way, Laindon, Basildon, SS15 6TP, Essex, United Kingdom (e-mail: vero.rotil@gmail.com).
\end{abstract} \\ VCOT Open 2022;5:e6-e10.
}

\begin{abstract}
Keywords

- bicipital tenosynovitis

- shoulder pain

- bony exostosis

- mineralization

- ossification

This report is of an osseous metaplasia of the transverse humeral ligament and secondary bicipital tenosynovitis in a 10-year-old Bengal cat. Computed tomography revealed the presence of an osseous bridge enclosing the bicipital groove, with ultrasonography confirming partial tear and inflammation of the bicipital tendon. Initial conservative management with intra-articular long-acting steroids failed to provide long-term improvement of clinical signs. Surgical excision of the osseous lesion resulted in complete resolution of lameness after surgery, confirmed by a 1-year followup. Histopathology of this lesion confirmed a well-differentiated osseous metaplasia of the transverse humeral ligament.
\end{abstract}

\section{Introduction}

The transverse humeral ligament (retinaculum) runs from the lesser to the greater tubercle of the humerus, preventing luxation of the biceps tendon from the intertubercular groove, and it is a well-known anatomical structure in the dog and cat. ${ }^{1,2}$ Gleason and colleagues ${ }^{3}$ concluded that in humans this structure was not an actual ligament, but rather a sling formed mainly by fibres of the subscapularis tendon, with contributions from the supraspinatus tendon and the coracohumeral ligament. Complete ossification of the transverse humeral ligament was described in a human cadaveric study in only one specimen. ${ }^{4}$ Clinical identification of mineralization of this structure in humans is limited to a sonographic study of the biceps tendon in less than $1 \%$ of shoulders studied. ${ }^{5}$ Calcification of the transverse humeral ligament and clinical manifestation of this have not been reported in the veterinary literature. This is the first case report of osseous metaplasia of the transverse humeral ligament in a cat treated surgically with a successful outcome.

received

August 26, 2021

accepted after revision

November 22, 2021
DOI https://doi.org/

$10.1055 / \mathrm{s}-0042-1742472$

ISSN 2625-2325.

\section{Case Description}

\section{History and Clinical Findings}

A 10-year-old, neutered, male Bengal cat weighing $4.5 \mathrm{~kg}$ was presented to the University of Liverpool Small Animal Teaching Hospital with a 1-month history of waxing and waning right thoracic limb lameness. No specific traumatic event was witnessed by the owner to trigger this lameness, which appeared to be more noticeable after exercise. Meloxicam $(0.05 \mathrm{mg} / \mathrm{kg}$, per os [PO], q24h) was administered for 2 weeks prior to presentation with no appreciable improvement. The patient was being medically managed for hypertrophic obstructive cardiomyopathy with benazepril hydrochloride/spironolactone $(0.25 / 2 \mathrm{mg} / \mathrm{kg}, \mathrm{PO}, \mathrm{q} 24 \mathrm{~h})$. On subjective gait analysis, a grade 4/10 right thoracic limb lameness ${ }^{6}$ was elicited. Orthopaedic examination was limited due to the irritable nature of the cat; however, a consistent pain response upon flexion of the right shoulder and concurrent pressure over the bicipital groove could be identified. Neurological examination was generally unremarkable.

\section{(c) 2022. The Author(s).}

This is an open access article published by Thieme under the terms of the Creative Commons Attribution License, permitting unrestricted use, distribution, and reproduction so long as the original work is properly cited. (https://creativecommons.org/licenses/by/4.0/) Georg Thieme Verlag KG, Rüdigerstraße 14, 70469 Stuttgart, Germany 


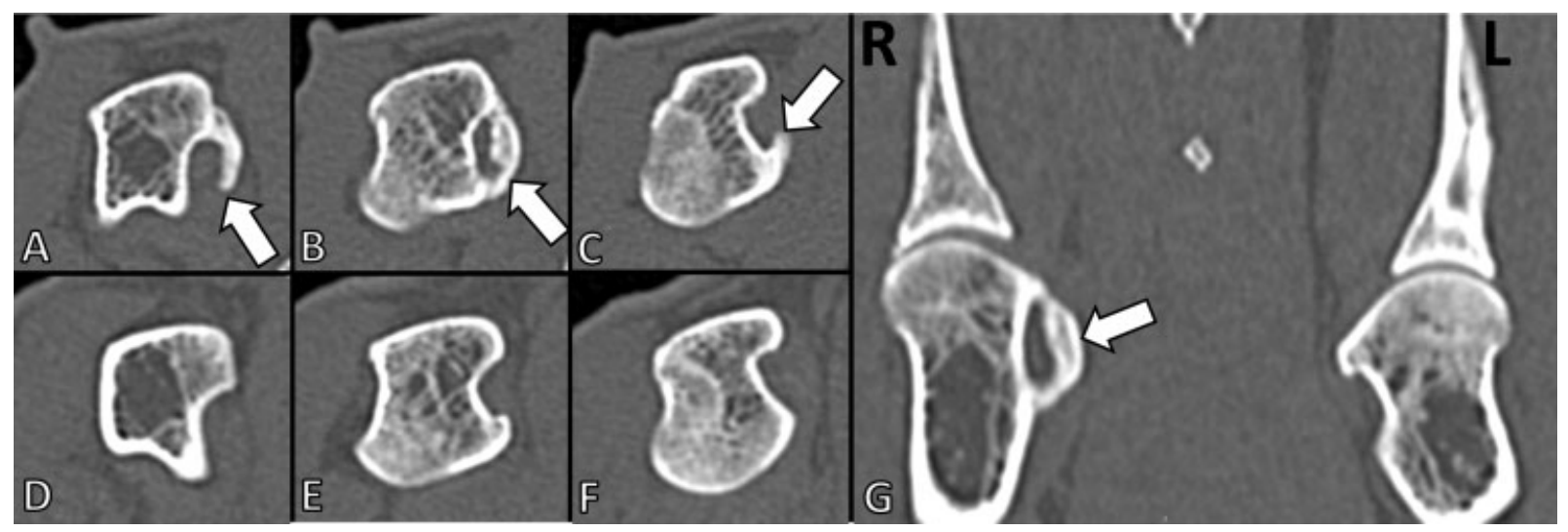

Fig. 1 (A-C) Transverse computed tomographic images from distal (A) to proximal (C) through the proximal right humerus of a 10-year-old Bengal cat. Note the curvilinear bone exostosis (arrowed) arising from the greater tubercle that fully encircles the biceps tendon in (B). (D-F) Transverse images of the proximal left humerus of the same cat at corresponding locations. (G) A dorsal plane reconstruction of the left and right scapulohumeral joints; the bone exostosis is arrowed. All images are sharp (bone) reconstructions displayed in a bone widow.

\section{Diagnostic Findings}

The patient was sedated and positioned in sternal recumbency for computed tomography (CT) of its thoracic limbs using an 80-slice multidetector CT scanner (Aquilion Prime, Toshiba Medical Systems, Tokyo, Japan). Reconstructions were generated using standard (soft tissue) and sharp (bone) kernel algorithms with a slice thickness of $0.5 \mathrm{~mm}$ and viewed in standard bone and soft tissue windows. The carpi and elbow joints were unremarkable. A well-defined curvilinear bony exostosis was identified projecting from the distal part of the greater tubercle of the right shoulder, which focally and completely encircled the proximal biceps tendon (-Fig. 1). The exostosis was smoothly marginated with no periosteal reaction. Mild osteophyte formation was apparent at the margins of the scapulohumeral joint (-Fig. 2). The left scapulohumeral joint was unremarkable.

Ultrasonography of the shoulder region confirmed the presence of a well-defined mineralized structure superficial to the right bicipital groove creating a distal acoustic shadow. A moderate volume of anechoic fluid was present surrounding

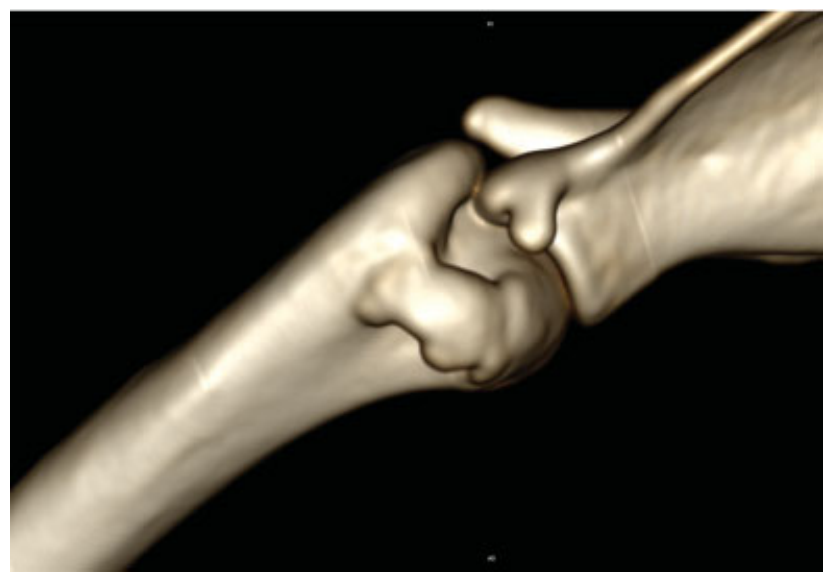

Fig. 2 Three-dimensional computed tomography reconstruction of the glenohumeral joint showing a curvilinear bony exostosis joining the greater and lesser tubercles of the right humerus. Proximal is to the right and distal to the left of the image. the tendon within the bicipital tendon sheath ( - Fig. 3). These findings were consistent with right biceps tenosynovitis.

\section{Management and Outcome}

Meloxicam was discontinued for 8 days prior to re-admission to the hospital. The patient was sedated with a combination of medetomidine $(0.04 \mathrm{mg} / \mathrm{kg}$, intramuscular [IM]), midazo$\operatorname{lam}(0.2 \mathrm{mg} / \mathrm{kg}, \mathrm{IM})$ and ketamine $(2 \mathrm{mg} / \mathrm{kg}$, IM) and placed in left lateral recumbency. The lateral aspect of the right shoulder was clipped and aseptically prepared. Arthrocentesis of the right shoulder using a 32-mm $23 \mathrm{G}$ hypodermic needle was performed, which yielded a mildly increased amount of macroscopically normal joint fluid. An in-house cytology revealed a mild increase in number of mononuclear cells (3-4 cells per high power field). Methylprednisolone acetate $(4.4 \mathrm{mg} / \mathrm{kg}$, intra-articular, once) was instilled into

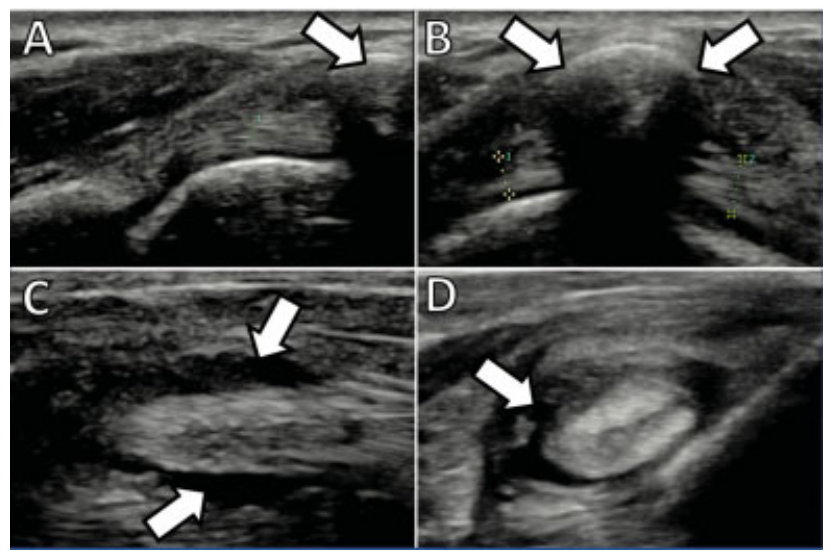

Fig. 3 Ultrasound images of the proximal brachial region of a 10-yearold Bengal cat. (A-C) Images aligned with the longitudinal axis of the bicipital tendon with proximal to the left; $(\mathrm{A})$ is the most proximal and (C) is the most distal. (D) is a transverse image of the tendon at approximately the same level as image $(C)$ with medial to the left. The biceps tendon is marked with measuring callipers in (A) and (B). Note the hyperechoic structure casting a distal acoustic shadow and obscuring the tendon (arrowed in A and B) and the effusion surrounding the biceps tendon (arrowed in $\mathrm{C}$ and $\mathrm{D}$ ). 
the right glenohumeral joint. The patient was discharged with instructions on strict cage rest.

Re-assessment after 4 weeks revealed an initial improvement of the lameness for approximately 3 weeks, with recurrence of the lameness after this period. At this stage, a grade 3/10 right thoracic limb lameness ${ }^{6}$ was still present and pain was elicited on manipulation of the right shoulder. Surgical management was discussed with the owner due to the only short-term relief of clinical signs with conservative management, reiterating the lack of evidence on outcome after surgical treatment.

Five weeks after steroid injection, the patient was anaesthetized and placed in left lateral recumbency. A craniomedial approach to the right glenohumeral joint was performed through a $5-\mathrm{cm}$ skin incision. ${ }^{7}$ The deltoideus muscle was identified and an incision made on the brachiocephalicus muscle elevating it from the proximal humerus. The thoracic limb was externally rotated and the superficial and deep pectoral muscles were partially elevated from the humerus. The area of ossification was identified over the tendon of biceps brachii muscle. This area was subsequently demarcated with four $25 \mathrm{G}$ hypodermic needles and a $1-\mathrm{mm}$ round-tip high-speed burr was used to remove the calcified structure. After this was elevated less than $50 \%$ of the fibres of the transverse humeral ligament were unaffected and left intact. During manipulation of the shoulder, the biceps tendon was not luxating and was sliding normally in the intertubercular groove even after challenging shoulder joint motion. The pectoral and brachiocephalic muscles were sutured in two separate simple continuous layers of three metric polydioxanone (PDS II, Ethicon). The subcutis and cutaneous layers were closed routinely, and the patient was discharged the following day with a 2-week course of meloxicam $(0.05 \mathrm{mg} / \mathrm{kg}, \mathrm{PO}, \mathrm{q} 24 \mathrm{~h})$ and further exercise restriction. Histopathology of the calcified tissue confirmed a welldifferentiated bone formation that effaced normal ligament collagen fibres, with a transition between fibrous dense tissue to bone structure which included osteoblasts, osteo-

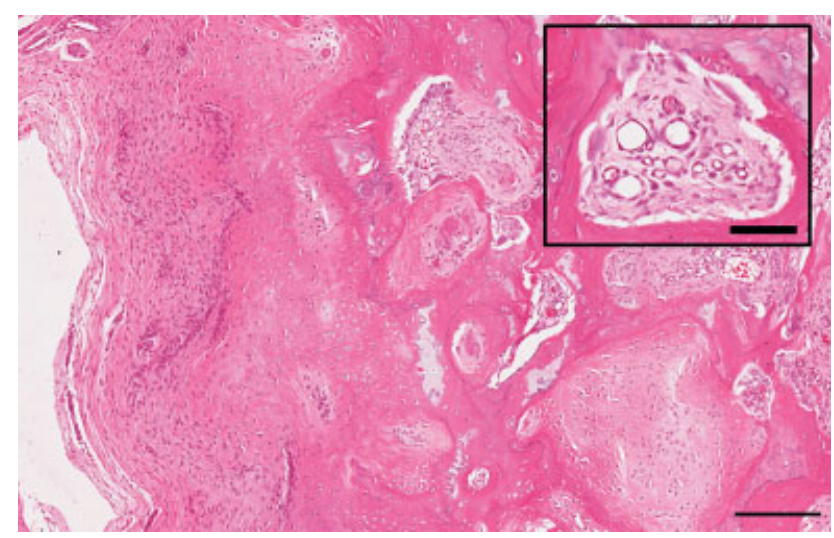

Fig. 4 Histology of the humeral ligament. There is a transition between normal tendon structure (left) and progressive osseous metaplasia (haematoxylin and eosin, decalcified specimen; scalebar = 200 microns). Inset: Higher magnification: evidence of formation of medullary cavities with osteoblasts and osteoclasts and blood vessels (haematoxylin and eosin, decalcified specimen; scalebar $=60$ microns). clasts and osteocytes and medullary cavities with blood vessels (-Fig. 4). This was classified as a well-differentiated osseous metaplasia of the transverse humeral ligament.

At follow-up appointment 8 weeks postoperatively, the cat was reported to have normal ambulation without noticeable lameness at home, and being able to jump onto furniture during the previous week without issues. No lameness could be elicited on subjective gait analysis and right shoulder manipulation was non-painful with a normal range of motion. Further imaging of the area was offered at this point and 1 year after surgery; however, the owner declined due to full clinical recovery and the potential risk of a further sedation procedure. On a telephone conversation with the owner 12 months after surgery the cat was reported to ambulate and jump normally with no identifiable lameness.

\section{Discussion}

This case of osseous metaplasia of the transverse humeral ligament seems to be the first reported in the veterinary literature. Limited information is available from the human literature, where this condition was reported on autoptic specimen ${ }^{4}$ and in a sonographic study in man where in four cases mineralization of this structure was identified, with concurrent bicipital tenosynovitis. ${ }^{5}$

Bicipital tenosynovitis is a common cause of shoulder pain in humans ${ }^{8}$ and in active, middle-aged or older, medium- to large-breed dogs. ${ }^{9}$ In cats, it is only described in one case report and was believed to be secondary to increased joint laxity associated with glenohumeral dysplasia and incongruity. ${ }^{10}$ In the case we present here, bicipital tenosynovitis revealed to be the cause of lameness as confirmed by the inflammation of the tendon on ultrasonographic assessment. This was presumed to be secondary to chronic chafing against the osseous bridge during multidirectional movements such as jumping, playing and climbing as demonstrated by the clinical improvement after removal of the osseous bridge. This chronic friction was believed to cause inflammation and microtearing that could eventually lead to degenerative changes in the tendon of the biceps, if untreated. Unfortunately, we were unable to prove this with postoperative imaging of the shoulder joint and biceps tendon due to the pet owner's denial.

In humans, genetic predisposition to calcification of other ligaments such as the superior transverse scapular ligament has been postulated from a familial case that affected father and son resulting in suprascapular nerve entrapment. ${ }^{11}$ Other aetiologies such as repetitive strain on the subscapularis, supraspinatus muscles and pectoralis major muscle, could result in microtrauma during biomechanical movements of the shoulder and ultimately lead to calcification and ossification of these fibres. ${ }^{4}$ There is limited information regarding osseous metaplasia of other ligaments in cats. A cadaveric feline study found similar metaplastic changes affecting the annular ligament in several cats that was postulated to be a result of mechanical (tensile) stress. ${ }^{12}$ A similar etiopathogenesis can only be speculated in the case reported here; however, 
no comparable lesions were identified on the thoracic limb joints of the same patient.

Thoracic limb lameness investigations in small animals have evolved in the past years with the advent of advanced imaging modalities such as CT. Recent studies have shown the increased sensitivity and specificity of CT for the detection of elbow and shoulder pathologies, ${ }^{13,14}$ with the addition of further information from CT arthrography in some shoulder pathologies. ${ }^{15}$ An initial CT scan was elected for this case to characterize the thoracic limb lameness and once pathology was confirmed in the shoulder, ultrasound of the bicipital groove and tendon was considered the imaging modality of choice due to its non-invasive nature and reliability in characterizing pathology of these structures. ${ }^{5,8,9}$ Despite shoulder arthroscopy being the gold standard diagnostic procedure for shoulder pathology, this would be unlikely to provide any further information to the case due to inability to directly observe the transverse humeral ligament. $^{2}$

Medical management of bicipital tenosynovitis has been successfully implemented, especially as a first-line treatment in dogs, $, 9,16$ involving the use of non-steroidal anti-inflammatory drugs, rest, physical therapy and intra-articular injection of long-acting steroids. Medical management by intra-articular corticosteroid administration was initially elected as previously recommended ${ }^{17}$ due to the unreported nature of the condition and unknown surgical outcome. There is continued controversy on the balance of beneficial or deleterious effects of intra-articular corticosteroids on articular cartilage and the overall joint environment; however, a systematic review suggested that there is not enough evidence about the effects of corticosteroids on cartilage and inflammation. ${ }^{18}$ In our case, this management failed to provide a long-term resolution of lameness, which was attributed to the continuous presence of the inciting cause. Therefore, surgical removal of the osseous bridge was elected in this case and resulted in a successful outcome. Surgical removal of the transverse humeral ligament has been performed with good success in cases of canine bicipital tenosynovitis ${ }^{9}$ however, other surgical options such as tenotomy or tenodesis have also been reported in dogs with equally good results. ${ }^{9,16,19}$ In our case, once the ossified lesion was removed, we could grossly identify some transverse fibres left in the region of the transverse humeral ligament, which could suggest that the transverse humeral ligament in cats is also formed of fibres originating from different tendons and ligaments of the area as it has been shown in humans ${ }^{3}$ however, this cannot be concluded without a dedicated anatomical study and could also be explained by partial mineralization of the transverse humeral ligament.

\section{Conclusion}

This is the first case report of osseous metaplasia of the transverse humeral ligament resulting in bicipital tenosynovitis in a 10-year-old Bengal cat. Diagnosis of the condition was achieved through orthopaedic examination, CT of the thoracic limbs and ultrasonography of the bicipital groove.
Conservative treatment failed to provide long-term resolution of lameness, with surgical excision of the osseous lesion resulting in fast resolution of clinical signs, as followed up 12 months after surgery.

\section{Authors' Contribution}

V. Rodiño Tilve, BVSc, GPCert, PgC(SAS), was involved in conception of the study, acquisition and interpretation of data, drafting of the manuscript, revision and approval of the submitted manuscript. T. Maddox, BVSc, PhD, CertVDI, DipECVDI, and L. Ressel, DVM PhD, DipECVP, FHEA, acquired and interpreted data, drafted the manuscript, revised and approved the submitted manuscript. R. Pettitt, BVSc, PGCertLTHE, DSAS(Orth), SFHEA, FRCVS, was involved in the conception of the study and study design, acquisition and interpretation of data, revision of the manuscript and approval of the submitted manuscript. All authors are publicly accountable for the relevant content of this report.

\section{Disclosure Statement}

The authors declare no conflict of interest related to this report.

\section{Funding}

None.

\section{References}

1 Evans HE, de Lahunta A. Miller's Anatomy of the Dog. 4th edition St. Louis, Missouri: Elsevier Saunders; 2013:168-169

2 Ridge P. Feline shoulder arthroscopy using a caudolateral portal, a cadaveric study. Vet Comp Orthop Traumatol 2009;22(04):289-293

3 Gleason PD, Beall DP, Sanders TG, et al. The transverse humeral ligament: a separate anatomical structure or a continuation of the osseous attachment of the rotator cuff? Am J Sports Med 2006;34 (01):72-77

4 Singh R. Analysis of bony bridge over bicipital groove. OA Case Rep 2013;2(13):1-3

5 Wurnig C. [Sonography of the biceps tendon]. Z Orthop Ihre Grenzgeb 1996;134(02):161-165

6 Dunning D. Orthopedic Rehabilitation [Internet]. [cited $2021 \mathrm{Sep}$ 26]. Accessed December 23, 2021 from: https://cvm.ncsu.edu/ wp-content/uploads/2015/06/Dunning-September2012VMF.pdf

7 Johnson KA. Piermattei's Atlas of Surgical Approaches to the Bones and Joints of the Dog and Cat. Fifth edition. St. Louis, Missouri: Elsevier Saunders; 2014:142-147

8 Farin PU, Jaroma H. The bicipital groove of the humerus: sonographic and radiographic correlation. Skeletal Radiol 1996;25 (03):215-219

9 Bruce WJ, Burbidge HM, Bray JP, Broome CJ. Bicipital tendinitis and tenosynovitis in the dog: a study of 15 cases. N Z Vet J 2000;48 (02):44-52

10 Scharf G, Steinbüchl S, Messmer M, Ohlerth S. Glenoid dysplasia and bicipital tenosynovitis in a Maine coon cat. J Small Anim Pract 2004;45(10):515-520

11 Cohen SB, Dines DM, Moorman CT. Familial calcification of the superior transverse scapular ligament causing neuropathy. Clin Orthop Relat Res 1997;(334):131-135

12 Breit S, Künzel W, Alton K, Kneissl S, Mayrhofer E. [Radiologically detectable changes in the radial annular ligament and the supinator muscle of the domestic cat (Felis catus)]. Schweiz Arch Tierheilkd 1998;140(06):231-239 
e10 Osseous Metaplasia of the Transverse Humeral Ligament in a 10-Year-Old Bengal Cat Rodiño Tilve et al.

13 Villamonte-Chevalier A, van Bree $\mathrm{H}$, Broeckx B, et al. Assessment of medial coronoid disease in 180 canine lame elbow joints: a sensitivity and specificity comparison of radiographic, computed tomographic and arthroscopic findings. BMC Vet Res 2015;11 (01):243

14 Maddox TW, May C, Keeley BJ, McConnell JF. Comparison between shoulder computed tomography and clinical findings in 89 dogs presented for thoracic limb lameness. Vet Radiol Ultrasound 2013;54(04):358-364

15 Eivers CR, Corzo-Menéndez N, Austwick SH, et al. Computed tomographic arthrography is a useful adjunct to survey computed tomography and arthroscopic evaluation of the canine shoulder joint. Vet Radiol Ultrasound 2018;59(05):535-544
16 Stobie D, Wallace LJ, Lipowitz AJ, King V, Lund EM. Chronic bicipital tenosynovitis in dogs: 29 cases (1985-1992). J Am Vet Med Assoc 1995;207(02):201-207

17 Piermattei D, Flo G, Decamp C. Brinker, Piermattei, Flo's Handbook on Small Animal Orthopedics and Fracture Repair. Fourth edition St. Louis, Missouri: Elsevier Saunders; 2006:289-290

18 Vandeweerd JM, Zhao Y, Nisolle JF, et al. Effect of corticosteroids on articular cartilage: have animal studies said everything? Fundam Clin Pharmacol 2015;29(05):427-438

19 Bergenhuyzen ALR, Vermote KAG, van Bree H, Van Ryssen B. Longterm follow-up after arthroscopic tenotomy for partial rupture of the biceps brachii tendon. Vet Comp Orthop Traumatol 2010;23 (01):51-55 